\title{
16 Yasmina Reza: der ,art de l'implicite‘ als ,art du carnage‘
}

In seiner Histoire de la conversation spricht Emmanuel Godo davon, dass die französische Konversation im 20. Jahrhundert kein Ideal mehr darstelle und den Gesichtspunkt der optimalen Verständigung aus dem Auge verloren habe. Stattdessen habe ein grundsätzlich konfliktuöses Verhalten die Überhand gewonnen. Die Konversation sei keine Kunst mehr, sondern spiegele das defekte Artikulationsvermögen der Zeitgenossen, welches eine der maßgeblichen Ursachen der Konflikte sei:

La conversation ne se pense plus comme un idéal mais comme une pratique révélant le malentendu indépassable qui fausse la connaissance de soi et biaise tout rapport à l'autre. La conversation ne se définit plus comme un art mais comme le miroir implacable de nos insuffisances. L'accord harmonieux cède la place à la relation conflictuelle et la conversation tourne à vide: on parle mais la parole n'est plus capable de faire un pont viable entre soimême et autrui. La conversation atteste désormais de la difficulté de dire et de se dire. ${ }^{1}$

Godos Beobachtungen sind Gegenstand des Theaters von Yasmina Reza. Die Autorin zeigt, wie konfliktuös eine Konversation aufgrund ihrer nach und nach zutage tretenden impliziten Dimensionen verlaufen kann. Das ästhetische Gefallen für den Zuschauer entsteht dabei dadurch, dass er an der Entwicklung der Gesprächssituation teilhat und nach und nach die impliziten Dimensionen der Äußerungen erkennt. Nathalie Sarrautes Ziel war es spürbar zu machen, inwieweit die ,sousconversations“ die offene Kommunikation stören. Yasmina Reza geht darüber hinaus. Sie lässt den Zuschauer ihrer Theaterstücke nicht nur die Tropismen der Figuren spüren sondern zugleich nachvollziehen, wie diese entstehen und wie sie die Akteure in ihrer Identität auflösen.

Bereits das erste Theaterstück Conversations après un enterrement, mit dem Reza 1986 debütierte, lässt erkennen, dass die Kommunikation im Zentrum ihrer Interessen steht. Die sechs Personen, die sich nach der Beerdigung von Simon Weinberg im Garten seines Hauses versammeln, ergründen in einer erkennbar an Sartres Stück Huis clos (1944) angelehnten Situation im Gespräch ihre Geheimnisse. Wenn der Sohn des Verstorbenen Alex seine Agressionen an Julienne, der dritten Ehefrau seines Onkels Pierre sowie an Elisa auslässt, die ihn aus Liebe zu seinem Bruder Nathan verlassen hat, dann bestätigt sich einmal mehr Sartres Er-

1 Une Histoire, S. 323.

2 Open Access. (c) 2021 Michael Bernsen, publiziert von De Gruyter (c))BY-NC-ND Dieses Werk ist lizenziert unter der Creative Commons Attribution 4.0 Lizenz.

https://doi.org/10.1515/9783110758009-016 
kenntnis: „L'enfer c'est les autres“2. Das Stück zeigt, wie die Familienmitglieder sich im Laufe des Gesprächs verändern, wie sich ihre Vorstellungen und Werte ungewollt verschieben ${ }^{3}$.

In Rezas Theaterstück Le Dieu du carnage, im Jahr 2006 verfasst und 2008 zum ersten Mal aufgeführt, werden solche Konversationen in ihrer Entstehung sichtbar und durch Reflexionen der Figuren sowie die Aufmerksamkeit des $\mathrm{Zu}$ schauers lenkende literarische Verfahren der Autorin fassbar gemacht. Zwei Ehepaare treffen sich, um einen Streit zwischen ihren beiden Söhnen zu schlichten. Der Sohn von Anette und Alain Reille hat dem Sohn von Véronique und Michel Houillé mit einem Stock zwei Zähne ausgeschlagen. Das Stück beginnt damit, dass die Familie Houillé eine Erklärung des Vorgangs aufsetzen will, die beide Ehepaare unterzeichnen sollen. Von vorneherein geht es dabei um die Macht der Worte und ihre Implikationen. Als es in der Erklärung heißt, Ferdinand Reille sei mit einem Stock bewaffnet gewesen („Ferdinand Reille, onze ans, armé d'un bâton“4), meldet der Vater sogleich Widerstand gegen diese Formulierung an, da ihm die seinen Sohn kriminalisierende Metapher zu weit geht. Die beiden Frauen versuchen, vermittelnd und besänftigend einzugreifen. Ihr Gespräch bringt das eigentliche Thema des Stücks auf den Punkt: Véronique Houillé bedankt sich bei den Reilles für ihr Kommen mit dem Satz: „On ne gagne rien à s'installer dans une logique passionnelle“ (S. 10). Und sie fährt mit der Einverständnis erheischenden Frage fort: „Par chance il existe encore un art de vivre ensemble, non?” (Ebd.)

Das Stück wird genau dies zur Diskussion stellen: Kann die Unterhaltung der beiden Ehepaare nach dem Prinzip des „art de vivre ensemble“ ohne „logique passionnelle“ vonstatten gehen? Gibt es überhaupt noch einen solchen ,art de vivre ensemble‘, wie er die französische Kommunikation über mehrere Jahrhunderte hinweg geprägt hat? Gibt es noch ,bienséances‘, die eine Gesprächssituation wie die vorliegende für alle verbindlich regulieren? Die unmittelbar folgende höfliche, scheinbar mitfühlende Nachfrage Anette Reilles, wie es um den abgebrochenen Zahn von Bruno Houillé bestellt sei, nutzt die Autorin, um dem Stand der ,conversio‘ und seinem , art de vivre ensemble“ eine implizite sinnbildliche Dimension zu verleihen: Es heißt, der Nerv des Zahns liege noch nicht blank („Ap-

2 Jean-Paul Sartre, Huis clos suivi de Les Mouches (Collections Folio. 807), Paris, Gallimard, 2000 (1972), S. 93.

3 Zur Analyse des Stücks vgl. Montserrat Serrano Mañes, „Conversations après un enterrement: l'exquise obscénité de Yasmina Reza et sa ,stratégie des poireaux““, Studi francesi Bd. 57/2013, H. 170, S. 416-423.

4 Yasmina Reza, Le Dieu du carnage, hrsg. von Sylvie Coly (Classiques \& contemporains. 128), Paris, Magnard, 2011 ( ${ }^{1}$ Paris, Albin Michel, 2007), S. 9. Die Stellenangaben im laufenden Text beziehen sich auf diese Ausgabe. 
paremment le nerf n'est pas complètement exposé“, ebd.) und man versuche, ihn zu erhalten („[...] pour le moment, on ne dévitalise pas“, ebd.). Bis dahin werde man den Zahn mit Keramik verkleiden („En attendand, il va avoir des facettes en céramique“, S. 11). Übersetzt bedeutet dies für den Zuschauer: Die Geschichte des Zahns wird zum Sinnbild der Unterhaltung der Kontrahenten. Noch bewegt diese sich auf der Ebene des zivilisierten Umgangs, noch ist die Konversation gemäß dem ,art de vivre ensemble، am Leben. So wie der teilweise blank liegenden Nerv des angegriffenen Zahns durch die Keramikverblendung wird die ,logique passionelle، im Gespräch durch ziviles Verhalten verdeckt. Diese Allegorien durch die Autorin sind eine Aufforderung an den Leser, über die indirekten, impliziten Dimensionen des Gesprächs nachzudenken. Rezas Stück ist nicht nur eine streitige Konversation; es ist auch eine Reflexion der ,sous-conversations“ dieser Unterhaltung.

Das Spiel der Autorin mit Implikationen des Gesagten wird im weiteren Verlauf des Stücks massiv vorangetrieben. Es zeigt dem Zuschauer, wie sich nach und nach die ,logique passionnelle‘ entwickelt, die das Gespräch zu dem im Titel angekündigten Gemetzel entarten lässt. Yasmina Reza setzt quasi Nathalie Sarrautes Beobachtung und Stilisierung von Tropismen fort. Sie tut dies über die Charakterisierung der Figuren: Alain Reille erhält permanent Anrufe auf seinem Handy, die er jeweils annimmt und auf diese Weise die gesamte Gesprächsrunde zwingt, daran teilzunehmen, was alle Beteiligten - insbesondere seine Gattin - zunehmend aggressiv macht. Bei diesen Anrufen geht es um seine anwaltliche Beratung einer pharmazeutischen Firma, die Probleme mit einem Blutdrucksenker hat, bei dem unerwünschte neurologische Nebenwirkungen auftreten. Diese Stilisierung des Alain Reille ist ein erneuter indirekter Hinweis der Autorin darauf, dass das Gespräch der Ehepaare nicht ohne eine Steigerung der Blutdruckwerte verlaufen wird. Auch die indirekte Charakterisierung der Figur der Véronique Houillé durch ihre Berufsbezeichnung lässt nichts Gutes erwarten: Wie Jean Racines Protagonistin Phèdre aus der gleichnamigen Tragödie, „fille de Minos et de Pasiphaé“, Tochter des Herrschers der Unterwelt und Enkelin des Sonnengottes Helios, Schatten und Licht ist, so ist auch sie - gleich auf mehrfache Weise - eine ,split personality': Sie ist Schriftstellerin und arbeitet nebenbei halbtags in einer „librairie d'art et d'histoire“. Der Leser konnotiert, dass sie einer kreativen Tätigkeit sowie ihrem Broterwerb nachgeht. Sie ist zuständig für die Kunst sowie die Realität, genau genommen - wie sich weiterhin zeigt - für die Zivilisation und den Terror: Publiziert hat sie nämlich einen Beitrag über die „civilisation sabéenne“, deren berühmte Königin der Legende nach bei König Salomon in Jerusalem in die Schule der Weisheit gegangen ist. Eine weitere Buchpublikation von ihr handelt von der Tragödie im sudanesischen Darfur, einem der blutigsten Bürgerkriege der neueren Zeit. Zwischen Zivilisation und Krieg wird sich die Konversation zwischen den 
Ehepaaren und den Ehepartnern im Verlauf des Stücks bewegen. Michel Houillé mit dem unspektakulären Beruf eines Großhändlers für Haushaltswaren ist dadurch charakterisiert, dass er abends zuvor den wegen seines ständigen Piepens nervtötenden Hamster der Tochter auf der Straße ausgesetzt hat. Und Anette Reille ist Beraterin bei der Verwaltung des Kulturerbes („,conseillère en gestion de patrimoine“, S. 14), wo sie sich doch später auf die geliebten Kunstbände der Familie Houillé erbricht, die dieses Kulturerbe im Bild aufbewahren.

Reza geht es mit der Unterhaltung der beiden Ehepaare nicht darum, eine kunstvolle, höflich geführte Unterhaltung vorzuführen, da sich die Teilnehmer meistens direkt äußern. Die Konversation führt vor, dass jede Äußerung, so direkt sie auch sein mag, indirekte Dimensionen haben kann und im konkreten Fall auch hat. Und diese indirekten Dimensionen erschließen sich nach und nach im Verlauf des Gesprächs. Mit jeder Äußerung geben die Figuren Auffassungen und Einstellungen preis, die ihre zuvor getätigten, direkten Aussagen in ein neues Licht rücken und die ,sous-conversations ‘ dieser Aussagen freilegen. D. h. Äußerungen erhalten im Nachhinein indirekte Dimensionen, weil sich Konnotationen einstellen, die sich aus Beobachtungen und Interpretationen ergeben. Im Zentrum von Rezas Gespräch steht somit der Aspekt, dass eine Konversation ein hoch dynamischer Vorgang ist: Es ergeben sich Veränderungen der Position eines Sprechers sowie der Wahrnehmung der Person durch die Beteiligten. Die indirekten Aspekte der Kommunikation ergeben sich erst allmählich.

Im Falle der Figur des Rechtsanwalts Alain Reille ist von vorneherein ersichtlich, dass er dem Gespräch nichts abgewinnen kann und will. Er ist von seiner Frau gezwungen worden teilzunehmen. Dementsprechend äußert er sich fortwährend im Modus der Ironie. Nachdem er Véronique Houillés ,clafoutis' gekostet und gelobt hat, bemerkt er ironisch: „Au moins ça nous permet de découvrir une recette." (S. 17). Seine telefonischen Einlassungen zu den Problemen des Blutdrucksenkers mit dem Verantwortlichen der pharmazeutischen Firma, die er vertritt, laufen vor allem darauf hinaus, der Gegenseite kein Recht zur Stellungnahme einzuräumen, da dies weitere Polemiken gegen das Mittel hervorrufen würde. Nicht nur diese Einlassungen veranschaulichen, dass es sich bei Alain um eine generelle Gesprächsverweigerung handelt, die in seiner Person begründet liegt und die auch den strittigen Fall seines Sohnes betrifft, über den er ja ebenfalls kein Gespräch führen will. Seine Einstellung zur Männlichkeit, so erklärt er Véronique, orientiere sich am Westerndarsteller John Wayne, für den, wie auch für ihn, die Konversation nicht als Problemlösung in Betracht komme: „Quand on est élevé dans une idée johnwaynienne de la la virilité, on n’a pas envie de régler ce genre de situation à coups de conversations.“ (S. 52). Alain vertritt die Position des Einzelkämpfers („,.... est-ce qu'on s'intéresse à autre chose qu’à soi-même?“, S. 55). für den allein das Recht des Stärkeren zählt. Als Rechtsanwalt sieht er Recht und 
Gesetz als einen Ersatz des ursprünglich auf der Gewalt beruhenden Rechts des Stärkeren: „Il faut un certain apprentissage pour substituer le droit à la violence. À l'origine je vous rappelle, le droit c'est la force.” (S. 61). Gegenüber Véronique bezeichnet er sich als Anhänger des ,Gottes des Gemetzels‘ und personifiziert auf diese Weise den Titel des Stücks. Per Andeutung lässt seine Formulierung darüber hinaus sogar rassistische Untertöne anklingen, und das an die Adresse von Véronique, die sich mit dem Krieg im afrikanischen Darfour beschäftigt hat: „,...] moi je crois au dieu du carnage. C'est le seul qui gouverne, sans partage, depuis la nuit des temps. Vous vous intéressez à l'Afrique n'est-ce pas... [...]” (S. 62). Alain ist somit erheblich für die Eskalation des Gesprächs verantwortlich. Sein Bekenntnis zum Recht des Stärkeren verhindert maßgeblich eine Kommunikation, die sich an ,bienséances“ orientiert und ein gewisses Maß an ,sociabilité‘ herstellt. Indirektes Sprechen, hier im Modus der Ironie, ist im Fall des Alain Reille Ausdruck einer ,sauvagerie‘, die er auch für seinen Sohn in Anspruch nimmt („,...] notre fils est un sauvage [...]“, S. 22). Seine amoralische Position untermauert er durch blasphemische Reden, bevor er beherzt zum Alkohol greift:

La morale nous prescrit de dominer nos pulsions mais parfois il est bon de ne pas les dominer. On n'a pas envie de baiser en chantant l'Agnus Dei. On le trouve ici ce rhum? (S. 64)

Für den Verlauf der Unterredung hat die zynisch-ironische Art des Sprechens von Alain allerdings eine wichtige Funktion: Während seine Frau ihm mehrfach vorwirft, zu nichts nutze zu sein (,Nous n'avons pas besoin de toi puisque tu ne sers à rien.“, S. 23) ist er es, der die hinter den Äußerungen der anderen stehenden strategischen Absichten entlarvt. Der Versuch, über ein Gespräch der Eltern die Wogen des Konflikts zu glätten, steht somit von vorneherein unter keinem guten Stern.

Die mangelnde Verständigungsbereitschaft Alains versuchen die drei anderen Beteiligten zunächst durch ein hohes Maß an Höflichkeit und zivilisierten Verhaltens zu kompensieren. Insbesondere die beiden Gastgeber, Michel und Véronique Houllié, stilisieren sich während des Gesprächs als Vertreter einer zivilisierten Form der Unterredung. Als Gegenpart zum stets zynischen Alain entpuppt sich dabei insbesondere Véronique. Sie beschwört nicht nur gleich zu Beginn den klassischen französischen , art de vivre ensemble‘. Auch während der Unterredung plädiert sie mehrfach für einen zivilen Umgang. Die von ihr gepriesene ,sociabilité‘ wird von Alain allerdings dahingehend enttarnt, dass sie auf lauter moralischen Geboten beruht:

Madame, il faudrait beaucoup de choses. Il faudrait qu'il vienne, il faudrait qu'il en parle, il faudrait qu'il regrette, vous avez visiblement des compétences qui nous font défaut, nous allons nous améliorer mais entre-temps soyez indulgente. (S. 24) 
Véroniques Einlassungen zielen immer wieder auf den Versuch, durch entsprechende Formulierungen den Sohn der Reilles zu diskreditieren und zu kriminalisieren. Nicht allein gegen die Formulierung „armé d'un baton“ wehrt Alain sich gleich zu Beginn; auch gegen ihre Äußerung, er habe seinen Mitschüler entstellt („,éfiguré son camarade“, S. 21) geht er an. Die Formel, man müsse Ferdinand als Verantwortlichen für die Tat ausmachen (,Si Ferdinand n'est pas responsabilisé [...]“, S. 23) stößt ebenso auf seinen Widerstand, wie der Versuch einer moralischen Verurteilung, Ferdinand habe Bruno „willentlich“ (,volontairement“, S. 29) geschlagen. Véronique wird auf diese Weise als genau jener Elterntyp enttarnt, der laut Anette völlig realitätsblind und ,infantil“ nur die eigenen Kinder im Blick hat: „Combien de parents prennent fait et cause pour leurs enfants de façon elle-même infantile.“ (S. 20). Die von Véronique eingangs propagierte Haltung, das Gespräch nicht in einer ,logique passionnelle‘ ausufern zu lassen, erweist sich als vorgetäuschte ,bienséance‘. Ihre moralische Attitüde, mit der sie auf die befriedende Wirkung der Kultur pocht (,Nous avons la faiblesse de croire aux pouvoirs pacificateurs de la culture.“, S. 25) bricht völlig in sich zusammen, nachdem die wahren, impliziten Absichten ihrer Äußerungen zu Tage treten bzw. von Alain ans Tageslicht gefördert werden. Geschult durch ihr Buch über den Darfour geht sie alsbald zum offenen Krieg über, bei dem alle zivilisatorischen Schranken fallen: „Puisque nous sommes modérés en surface, ne le soyons plus!“ (S. 44), heißt es. Und als die Reilles sich wieder einmal aus dem Gespräch verabschieden wollen, erklärt sie jedwede ,honnêteté für beendet:

Ça ne sert à rien de bien se comporter. L'honnêteté est une idiotie, qui ne fait que nous affaiblir et nous désarmer... (S. 46)

Véronique gerät zunehmend in ein Streit mit ihrem Ehemann, der seinerseits im Stil Alains zynische Bemerkungen zu ihren Überlegungen und moralischen Vorschriften macht:

VÉRONIQUE. Je ne sais pas ce que veut dire raisonner trop. Et je ne vois pas à quoi servirait l'existence sans une conception morale du monde.

MICHEL. Voyez ma vie!

VÉRONIQUE. Tais-toi! Tais-toi! J'exècre cette connivence minable! Tu me dégoûtes! (S. 56)

Als Michel mit ironischem Unterton den Provokateur Alain davor warnt, Véronique auf das Thema ,Darfour` zu bringen, beginnt sie auf ihren Ehemann einzuschlagen:

Ne la lancez pas là-dessus! Par pitié!

Véronique se jette sur son mari et le tape, plusieurs fois, avec un désespoir désordonné et irrationnel. (S. 63) 
Dieser erklärt, er sei ihres simplifizierenden moralisierenden „Humbugs“ überdrüssig und stellt sie auf eine Stufe mit den „Negern aus dem Sudan“:

MICHEL. Oh tu fais chier Véronique, on en a marre de ce boniment simpliste!

VÉRONIQUE. Que je revendique.

MICHEL. Oui, oui, tu revendiques, tu revendiques, ça déteint surtout maintenant ton engouement pour les nègres du Soudan. (S. 73)

Ähnlich wie bei Véronique werden auch die impliziten Einstellungen ihres Ehemanns Michel, die hinter seinen Äußerungen stehen, im Lauf der Unterredung deutlich. Wie seine Ehefrau tritt auch er zunächst besänftigend auf und plädiert für eine ,irritationsfreie“ Unterredung:

Nous sommes des gens de bonne volonté. Tous les quatre, j'en suis sûr. Pourquoi se laisser déborder par des irritations, des crispations inutiles?... (S. 44)

Seine Haltung gegenüber der befriedenden Wirkung der Kultur sind jedoch von vorneherein nicht so gefestigt, wie die seiner Ehefrau. So geht er davon aus, dass der Prozess der Zivilisierung eines Menschen ein langer Formierungsprozess ist, der - so seine Meinung - möglicherweise erst am Ende des Lebens seine Form gewinnt:

Moi je dis toujours, on est un tas de terre glaise et de ça il faut faire quelque chose. Peut-être que ça ne prendra forme qu’à la fin. (S. 15)

Als der eigentliche Grund für den Streit der beiden Söhne herauskommt, dass Bruno Houillé den Sohn der Reilles nicht in seine Bande aufnehmen wollte, bricht auch seine Fassade zivilen Verhaltens zusammen. Er erklärt, dass der Mensch nicht wirklich dauerhaft eine Selbstkontrolle ausüben könne: „Moi je dis, on ne peut pas dominer ce qui nous domine.“ (S. 36). Und er outet sich, als Jugendlicher selbst Bandenführer gewesen zu sein. Dies passt dann zu jener eingangs erwähnten Untat, den Hamster seiner Tochter auf der Straße auszusetzen. Darauf kommt Annette Reille mehrfach zurück, die sich in die Rolle der Schuldigen gedrängt sieht, wo doch Michel in ihren Augen selbst ein „Mörder“ sei:

Vous vous efforcez de nous culpabiliser, vous avez mis la vertu dans votre poche alors que vous êtes un assassin vous-même. (S. 47)

Michel enttarnt schließlich, inwieweit er und seine Frau den Rahmen für die Unterredung inszeniert und die Rollen festgelegt haben, und erklärt, er sei in Wirklichkeit ein Psychopath:

Je vais vous dire, toutes ces délibérations à la con, j'en ai par-dessus la tête. On a voulu être sympathiques, on a acheté des tulipes, ma femme m’a déguisé en type de gauche, mais la vérité est que je n'ai aucun self-control, je suis un caractériel pur. (S. 49) 
Den Verlust ihrer ,contenance“ hat auch die eher blasse Annette Reille zu verzeichnen. Ihre Strategie ist es, das Opfer des Streits Bruno Houillé als mitschuldig darzustellen. In ihren Augen ist die Ablehnung der Aufnahme ihres Sohnes Ferdinand in die Bande Brunos eine Beleidung, die eine Reaktion erfordert hat:

Ferdinand s'est fait insulter et il a réagi. Si on m’attaque, je me défends surtout si je suis seule face à une bande. (S. 44)

Damit übernimmt sie ein Stück weit die Positionen ihres zynischen Mannes Alain und dessen Ansicht, alle seien letzten Endes ,Wilde‘. Nach dem Genuss des ,clafoutis‘ muss sie sich erbrechen, was die Unterredung geradezu vulkanartig auf eine neue, nunmehr unzivilisierte Stufe der Auseinandersetzung hebt. Sie lässt letztlich jede Hemmung fallen, und gibt sich ungezügelt dem Genuss des von Michel angeboten Alkohols hin, mit dem Ergebnis einer völligen Entgleisung:

On vient dans leur maison pour arranger les choses et on se fait insulter, et brutaliser, et imposer des cours de citoyenneté planétaire, notre fils a bien fait de cogner le vôtre, et vos droits de l'homme je me torche avec! (S. 74)

Diese Entgleisung wird von Michel mit einem ironischen Kommentar bedacht: „Un petit coup de gnôle et hop le vrai visage apparaît. Où est passée la femme avenante et réservée, avec une douceur de traits...” (Ebd.)

Yasmina Rezas Dieu du carnage ist eine Positionsbestimmung, wie es um die Vorstellungen und Werte der französischen Konversation steht. Zentrale Begriffe der Traktate über die Konversation aus der Zeit der höfischen Kommunikation wie der ,art de bien vivre ensemble‘ oder die ,honnêteté‘ durchziehen das Stück. Sinnbild für das gesamte Stück wird schließlich am Schluss die Aussetzung des Hamsters auf der Straße: Als die Tochter der Houillés anruft, um sich nach der Befindlichkeit des Hamsters zu erkundigen, stellen sich die Fragen erneut, die sich bei seiner Aussetzung schon gestellt hatten: Ist der Hamster im Käfig glücklich („Tu penses qu'elle se plaisait dans une cage ?“, S. 78)? Kann er sich in der Freiheit überhaupt behaupten (,Grignote est très débrouillarde [...] elle est omnivore comme nous.“, ebd.)? Ist der Mensch, gefangen in den Konventionen des ,art de bien vivre ensemble‘ glücklich, oder wäre er befreit aus diesen Konventionen glücklicher? Die Autorin lässt diese Fragen bewusst offen :

MICHEL. Si ça se trouve, cette bête festoie à l'heure qu'il est.

VÉRONIQUE. Non.

Silence.

MICHEL. Qu'est-ce qu'on sait? (S. 78)

Nach Emmanuel Godo muss die Literatur diese Fragen stellen, weil die indirekte Kommunikation stets die Sprache der Literatur gesprochen hat und spricht. Wenn 
auch das klassische französische Modell der ,sociabilité‘ qua Konversation, das auf der Kunst des Impliziten beruht, in der Gegenwart nicht mehr die positiven Züge aufweist, wie in der Vergangenheit, dann ist es weiterhin die Aufgabe der Literatur, darüber Reflexionen anzustellen, wie und mit welchen Mitteln Kommunikation zustande kommt:

Elle qui se concevait, dans la tradition classique, comme un idéal de transmission, a pour vocation principale, dorénavant, de dévoiler l'opacité foncière qui entrave la connaissance que l'on peut avoir de soi et celle que l'on voudrait avoir des autres. La parole n'établit plus de lien: elle témoigne de l'impossibilité d'établir avec autrui un rapport sain. La conversation cesse de se concevoir comme un modèle de sociabilité et apparaît comme une zone de turbulences dans l'espace de laquelle se déploie la tragédie souvent burlesque de l'incommunicabilité. C'est pourquoi la conversation demeure, par-delà la faillite du modèle classique, un enjeu majeur des représentations, au premier rang desquelles la littérature. Artificielle, superficielle, source de frustrations, révélatrice autant de nos attentes que de notre incapacité, mi-subie, mi-volontaire, à les satisfaire, la conversation reste, pour la littérature du XX $\mathrm{X}^{\mathrm{e}}$ siècle, un objet capital. ${ }^{5}$

5 Une Histoire, S. 324. 
